\title{
THE ROLE OF TRAINING OR HINTS RELATING TO "THINKING IN OPPOSITES” IN INSIGHT PROBLEM SOLVING
}

\author{
Erika Branchini ${ }^{1}$, Roberto Burro ${ }^{1}$, Elena Capitani ${ }^{2}$, Ugo Savardi $^{1}$, \& Ivana Bianchi $^{3}$ \\ ${ }^{I}$ Department of Human Sciences, University of Verona (Italy) \\ ${ }^{2}$ Department of Education, Cultural Heritage and Tourism, University of Macerata (Italy) \\ ${ }^{3}$ Department of Humanities, Section of Philosophy and Human Sciences, University of Macerata (Italy)
}

\begin{abstract}
In recent years, there has been a growing desire to remove the mystery from insight, the underlying processes of which are not fully understood (Gilhooly et al., 2015). The present research focuses on the hypothesis that "thinking in opposites" might facilitate the process of representational change required for solving visuo-spatial insight problems (Bianchi et al., 2019; Branchini et al., 2015, 2016). There is evidence in Cognitive Science that opposites (or contrasts) are a central cognitive structure in space perception (e.g. Bianchi et al., 2017) and language (e.g. Jones et al., 2012) and they are fundamental to inductive (Gale and Ball, 2012) and deductive reasoning (Augustinova, 2008). They are also involved in creative thinking (Rothenberg, 2001).

In particular, the study reports the results of research carried out with the aim of investigating whether prompting problem solvers to use a strategy based on the manipulation of opposites (i.e. inside-outside, large-small) would improve their performance (Bianchi et al., 2019). Two hundred and forty undergraduate students were asked to analyze the spatial features inherent to six problems in terms of opposites before embarking on their search for a solution. Two hint and two training conditions were studied.

The results show that success rates increased when the participants were explicitly trained to use opposites.

In relation to the current debate on the factors which facilitate insight problem solving (e.g. Ahmed and Patrick, 2006; Cunningham and MacGregor, 2008; Patrick et al., 2015), our results add evidence that a prompt "to think in opposites" is a facilitating factor when it is given explicitly (i.e. as part of training). The findings are also discussed in relation to a debate on the same subject regarding the differences between the role of Type 1 processes (which are automatic, unconscious and associative) and that of Type 2 processes (which are controlled, conscious and analytical) (e. g. Weisberg, 2015, 2018). In particular, we provide suggestions concerning the nature of the role of opposites in the generation of potential solutions (Type 1 processes) and in the evaluation of these various potential alternatives with a view to homing in on a single solution (Type 2 processes).
\end{abstract}

Keywords: Insight problem solving, opposites, representational change, training versus hint, Type 1 versus Type 2 processes.

\section{Introduction}

Some problems appear trivial at a first glance, yet in reality finding their solution turns out to be very difficult and time-consuming. Despite the initial impression, there may not be an obvious solution to the problem and the application of strategies which are familiar to the problem solver (i.e. which have been used in the past to solve similar problems) are not necessarily successful. When the solution is finally found, it often appears suddenly and unexpectedly and is frequently accompanied by an "aha" experience. This is why they are known as "insight" problems (Öllinger and Knoblich, 2009). A representational change is needed in order to solve this type of problem (Ohlsson, 2011).

The way in which people tackle these problems is not fully understood and there has been a growing desire to remove the mystery surrounding them by means of theory driven experiments (Gilhooly et al., 2015). A question which remains open concerns whether there are any factors which may facilitate insight, either on an explicit or implicit level. This issue is related to the more general debate on the role of automatic and unconscious (Type 1) processes versus controlled and conscious (Type 2) 
processes in insight problem solving (Weisberg, 2015; 2018). While hints represent implicit suggestions or prompts (e.g. Bröderbauer et al., 2013 and on the efficacy of hints see for instance Bröderbauer et al., 2013), training programs explicitly teach problem solvers a strategy or procedure that can be recalled later on to solve problems with a similar structure (Patrick and Ahmed, 2014; on the efficacy of training procedures, see for instance Patrick et al., 2015). The study discussed in this paper (and presented in detail in Bianchi et al., 2019) delves deeper into this issue while specifically testing the role of "thinking in opposites" as a facilitating factor. Some studies have put forward the idea that the process of representational change required to solve visuo-spatial insight problems can be facilitated by "thinking in opposites" (Bianchi et al., 2019; Branchini et al., 2015; Branchini et al., 2016). However, what these studies did not clarify was whether a prompt to use opposites works better when proposed as an explicit strategy and systematically applied or as a hint acting at a less systematically-driven level.

\section{Design}

Four conditions were tested. These resulted from a 2x2 factorial experimental design with one factor being the Type of Facilitation (two levels: Hint and Training) and another factor the Identification of Opposite properties (two levels: Overt Listing and Covert Listing).

In the Training conditions, before the six visuo-spatial insight problems were presented to the participants, they were given a brief training session in which an experimenter used three example problems to demonstrate how a three step strategy based on the manipulation of spatial opposites might help them to solve the problems. The first step consisted of identifying all the spatial properties inherent to the problem (both in the written text and in the figure); the second step involved identifying the corresponding opposite for each property found in step one. In the third step they were asked to assess whether using these opposite properties might help them to find the solution. In one variant of the Training condition, the participants were asked to write down a list of the opposites identified in the second step (Overt Listing Training condition, OLT); in another condition, this was not required (Covert Listing Training condition, CLT).

In the Hint conditions, before the six problems were presented, the participants were told that transforming the spatial properties of the problem into their opposites would help them to find the solution. To this end, they were requested to identify all of the spatial properties inherent to the problem (both in the written text and in the figure) and determine their corresponding opposites before embarking on their search for the solution. There were two Hint conditions in this case too. In one condition (the Overt Listing Hint condition, OLH), the participants were asked to write down the list of opposites they had identified and in another condition (the Covert Listing Hint condition, $\mathrm{OCH}$ ), the participants were not asked to make an explicit list.

For demonstration purposes, three example problems were used both in the training and in the hint conditions. In all of the conditions, the participants were in small groups of three members. Previous studies had in fact proved the benefits of "contrast thinking" which arises when people work in groups rather than individually (Augustinova, 2008; Branchini et al., 2016).

\section{Objectives}

The aim of the study was to explore how different levels of awareness and systematicity pertaining to the use of opposites might affect visuo-spatial insight problem solving. The two types of facilitation tested (i.e. Hint versus Training) presupposed two different levels of awareness in the application of a strategy based on opposites. The two conditions relating to the identification of the opposites inherent to the problem (i.e. Overt Listing versus Covert Listing) stimulated different levels of systematicity when the participants manipulated the structure of the problem.

\section{Method}

Two hundred and forty undergraduate students at the University of Verona, Italy took part in the study (111 males, 129 females; $M_{\text {age }}=21.83$ years, $S D=7.26$ years) in small groups of three members. Sixty participants (i.e. 20 groups) were randomly assigned to each of the four conditions studied: the Overt Listing Training condition (OLT), the Covert Listing Training condition (CLT), the Overt Listing Hint condition $(\mathrm{OLH})$ and the Covert Listing Hint condition (CLH).

Six target problems were used with the order of presentation randomized between groups: the "pigs in a pen" problem (Schooler et al., 1993); the "triangle" problem (De Bono, 1969); the "deer" problem (origin unknown); the "eight-coin" problem (Ormerod et al., 2002); the "five-square" problem 
(Katona, 1940) and the "circumference" problem (Köhler, 1969). Three example problems were used in the instruction booklets in all of the training and hint conditions. These were the "parallelogram" problem and the "altar window" problem (both devised by Wertheimer, 1919/1945) and the "nine dot" problem (devised by Maier, 1930). The instructions were printed on sheets of paper given to the participants. These were read out by the experimenter who then answered any questions as necessary. The experimenter then handed out a booklet containing the six problems to be solved. Since a group problem solving task was foreseen, only one booklet per group was provided and the participants were repeatedly encouraged to talk aloud while trying to find the solution together. They were given seven and a half minutes for each problem.

\section{Discussion}

Two main aspects emerged from the results of the study (for further details and analysis, see Bianchi et al 2019; all data were analyzed using GLMM - Borenstein, Hedges, Higgins, \& Rothstein, 2009).

One aspect refers to success rates. The participants in the Training conditions solved a greater number of problems than the participants in the Hint conditions (main effect of Type of Facilitation: $\chi^{2}(1, \mathrm{~N}=80)=6.530, p=.011, z$-ratio $\left.=-3.993, d=-0.446\right)$. This suggests that explicitly teaching a strategy based on the manipulation of opposite features works better that simply giving a hint to think in terms of opposites.

The second aspect refers to the effect of overtly listing the opposites identified in the problem structure in the preliminary phase. Overt listing improved the participants' ability to solve the problems (i.e. they solved a greater number of problems) in the Hint condition but not in the Training condition (significant interaction between the Type of Facilitation and Identification of Opposites: $\chi 2{ }_{(1, \mathrm{~N}=80)}=4.453, p=.035$; post hoc OLH vs OLH: $S T=0.986, S E=0.354, z$-ratio $=2.781, p=0.03$, $d=0.311)$.

These results suggest that a certain degree of systematicity in the application of the prompt to think in opposites is needed. In the training condition, this systematicity seemed to have been sufficiently guaranteed by the three steps that the participants were instructed to follow (see the Design section). Conversely, in the hint condition, asking participants to write down the lists of opposites identified in the preliminary phase helped them to remain anchored to the manipulation of these characteristics when searching for the solution and this led to a better success rate. This anchoring effect emerged both from an analysis of the sketches done by the participants when they were searching for the solution and from the verbal dialogues between the members of each group. These analyses were possible since the sketches were collected after each session and video recordings were made during the sessions.

\section{Conclusion}

The findings reported in this article support previous evidence regarding the facilitating effect of "thinking in opposites" in visuo-spatial insight problem solving (Bianchi et al., 2019; Branchini et al., 2016; Branchini et al., 2015a, 2015b; Branchini et al., 2009). In particular they indicate that this strategy works more effectively when systematically applied, that is, when contextualized in an explicit procedure (in this case involving three steps) or when hinted at after an overt identification of the opposites to be focused on.

Various studies have shown that the restructuring of the problem which is a necessary part of the process is facilitated when problem solvers are given training during which it is revealed that some of the tacit assumptions they are making work as real constraints and prevent them from seeing the solution (Ahmed and Patrick, 2006; Cunningham and MacGregor, 2008; Patrick et al., 2015). Adopting a strategy of thinking in terms of opposites helps to overcome these constraints by suggesting the "obvious" direction which the transformation of the structure of the problem should take. We say "obvious" since intuitively the concept of opposites is very readily evoked by people (even at a very early age), despite the fact that invariably they are unable to give an explicit definition of the concept (Bianchi et al., 2011; Casasola, 2008; Casasola et al., 2003; Savardi and Bianchi, 2008). The "intuitivity" of the concept of opposites connects Opposites to Type 1 processes. Conversely, when Opposites provide the framework for a systematic revision of alternative possible manipulations, they seem to work more in terms of Type 2 (i.e. conscious) processes.

Evidence that opposites support visuo-spatial insight problem solving (Bianchi et al., 2019; Branchini et al., 2016; Branchini et al., 2015a, 2015b; Branchini et al., 2009) is in line with other studies in the field of Cognitive Science which have demonstrated the role of opposites in several human cognitive abilities. These range from perception (Bianchi et al., 2017) to the organization of conceptual 
spaces (Jones et al., 2012), to inductive (Gale and Ball, 2012) and deductive reasoning (Agustinova, 2008) and also to creative thinking (Rothenberg, 2001).

Two limitations of the present state of art of research on the benefits of thinking in terms of opposites in problem solving concern, firstly, the generalizability of these conclusions beyond visuo-spatial problem solving and, secondly, its generalizability beyond the group condition. Why is "thinking in opposites" associated with better performance when problem solvers are in small groups rather than working individually? One hypothesis is that when problem solvers work in groups, this facilitates "U-turns" in thinking processes and disconfirming strategies since it is easier to disconfirm the suggestions of others than one's own ideas (on confirmation biases see for instance Kuhn and Udell, 2007; Mercier and Sperber, 2011). In any case, further studies are needed to better understand whether there are any weaknesses in the "thinking in opposites" strategy when used by individuals in problem solving.

\section{References}

Ahmed, A., \& Patrick, J. (2006). Making implicit assumptions explicit in verbal insight problem solving. In Cognitive Science Society (Ed.), Proceeding of 28th Annual Conference of the Cognitive Science Society in Cooperation with 5th International Conference of Cognitive Science Society (pp. 986-991). Red Hook, NY: Curran Associates, Inc.

Augustinova, M. (2008). Falsification cueing in collective reasoning: Example of Wason selection task. European Journal of Social Psychology, 38 (5), 770-785.

Bianchi, I., Branchini, E., Burro, R., Capitani, E., \& Savardi, U. (2019). Overtly prompting people to "think in opposites" supports insight problem solving. Thinking \& Reasoning. Retrieved from: https://doi.org/10.1080/13546783.2018.1553738

Bianchi, I., Paradis, C., Burro, R., van de Weijer, J., Nyström, M., \& Savardi, U. (2017). Identification of opposites and intermediates by eye and by hand. Acta Psychologica, 180, 175-189.

Bianchi, I., Savardi, U., \& Kubovy, M. (2011). Dimensions and their poles: A metric and topological theory of opposites. Language and Cognitive Processes, 26 (8), 1232-1265.

Borenstein, M., Hedges, L. V., Higgins, J. P. T., \& Rothstein, H. R. (2009). Introduction to meta-analysis. London: Wiley.

Branchini, E., Bianchi, I., Burro, R., Capitani, E., \& Savardi, U. (2016). Can contraries prompt intuition in insight problem solving? Frontiers in Psychology, 7:1962.

Branchini, E., Burro, R., Bianchi, I., \& Savardi, U. (2015). Contraries as an effective strategy in geometrical problem solving. Thinking \& Reasoning, 21 (4), 397-430.

Branchini, E., Burro, R., \& Savardi, U. (2009). Contraries in productive thinking. In U. Savardi (Ed.), The Perception and Cognition of Contraries (pp. 203-224). Milan: McGraw-Hill.

Branchini, E., Savardi, U., \& Bianchi, I. (2015). Productive thinking: the role of perception and perceiving opposition. Gestalt Theory, 37, 7-24.

Bröderbauer, S., Huemer, M., \& Riffert, F. (2013). On the effectiveness of incidental hints in problem solving revisiting Norman Maier and Karl Duncker. Gestalt Theory, 35, 349-364.

Casasola, M. (2008). The development of infants' spatial categories. Current Directions in Psychological Science, 17, 21-25.

Casasola, M., Cohen, L. B., \& Chiarello, E. (2003). Six-month-old infants' categorization of containment spatial relations. Child Development, 74, 679-693.

Cunningham, J. B., \& MacGregor, J. N. (2008). Training insightful problem solving: Effects of realistic and puzzle-like contexts. Creativity Research Journal, 20, 291-296.

De Bono, E. (1969). The mechanism of mind. New York, NY: Penguin.

Gale, M., \& Ball, L. J. (2012). Contrast class cues and performance facilitation in a hypothesis testing task: Evidence for an iterative counterfactual model. Memory \& Cognition, 40, 408-419.

Gilhooly, K., Ball, L., \& Macchi, L. (2015). Insight and creative thinking processes: Routine and special. Thinking \& Reasoning, 21 (1), 1-4.

Jones, S., Murphy, M. L., Paradis, C., \& Willners, C. (2012). Antonyms in English: Construals, constructions, and canonicity. Studies in English language. Cambridge: Cambridge University Press.

Katona, G. (1940). Organizing and memorizing: Studies in the psychology of learning and teaching. New York, NY: Columbia University.

Köhler, W. (1969). The task of Gestalt psychology. Princeton, NJ: Princeton University Press. 
Kuhn, D., \& Udell, W. (2007). Coordinating own and other perspectives in argument. Thinking and Reasoning, 13, 90-104.

Maier, N. R. F. (1930). Reasoning in humans. On direction. Journal of Comparative Psychology, 10 (2), $115-143$.

Mercier, H., \& Sperber, D. (2011). Why do humans reasons? Arguments for an argumentative theory. Behavioral and Brain Sciences, 34, 57-111.

Ohlsson, S. (2011). Deep learning: How the mind overrides experience. Cambridge: Cambridge University Press.

Öllinger, M., \& Knoblich, G. (2009). Psychological research on insight problem solving. In H. Atmanspacher \& H. Primas (Eds.), Recasting reality (pp. 275-300). Berlin, Germany: Springer.

Ormerod, T. C., MacGregor, J. N., \& Chronicle, E. P. (2002). Dynamics and constraints in insight problem solving. Journal of Experimental Psychology: Learning, Memory \& Cognition, 28, 791-799.

Patrick, J., \& Ahmed, A. (2014). Facilitating representation change in insight problems through training. Journal of Experimental Psychology: Learning, Memory \& Cognition, 40, 532-543.

Patrick, J., Ahmed, A., Smy, V., Seeby, H., \& Sambrooks, K. (2015). A cognitive procedure for representation change in verbal insight problems. Journal of Experimental Psychology: Learning, Memory \& Cognition, 41, 746-759.

Rothenberg, A. (2001). Bipolar illness, creativity, and treatment. Psychiatric Quarterly, 72 (2), 131-147.

Schooler, J. W., Ohlsson, S., \& Brooks, K. (1993). Thoughts beyond words: When language overshadows insight. Journal of Experimental Psychology: General, 122 (2), 166-183.

Weisberg, R. W. (2015). Toward an integrated theory of insight in problem solving. Thinking \& Reasoning, 21, 5-39.

Weisberg, R. W. (2018). Problem solving. In L. J. Ball \& V. A. Thompson (Eds.), The Routledge International Handbook of Thinking and Reasoning (pp. 1239-1274). New York, NY: Routledge.

Wertheimer, M. (1945). Productive thinking. New York: Harper. First appearance Über Schulzbprozesse productiven Denken (Original work published in 1919). 